\title{
Article
}

\section{Solar energetic particle transport near a heliospheric current sheet}

Battarbee, Markus, Dalla, Silvia and Marsh, Mike S

Available at https://clok.uclan.ac.uk/19638/

Battarbee, Markus orcid iconORCID: 0000-0001-7055-551X, Dalla, Silvia orcid iconORCID: 0000-0002-7837-5780 and Marsh, Mike S (2017) Solar energetic particle transport near a heliospheric current sheet. The Astrophysical Journal, 836 (1). ISSN 0004-637X

It is advisable to refer to the publisher's version if you intend to cite from the work. http://dx.doi.org/10.3847/1538-4357/836/1/138

For more information about UCLan's research in this area go to

http://www.uclan.ac.uk/researchgroups/ and search for < name of research Group>.

For information about Research generally at UCLan please go to http://www.uclan.ac.uk/research/

All outputs in CLoK are protected by Intellectual Property Rights law, including Copyright law. Copyright, IPR and Moral Rights for the works on this site are retained by the individual authors and/or other copyright owners. Terms and conditions for use of this material are defined in the policies page.

\section{CLoK}

Central Lancashire online Knowledge www.clok.uclan.ac.uk

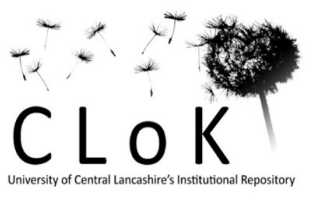




\title{
Solar Energetic Particle Transport Near a Heliospheric Current Sheet
}

\author{
Markus Battarbee ${ }^{1}$, Silvia Dalla ${ }^{1}$, and Mike S. Marsh ${ }^{2}$ \\ ${ }^{1}$ Jeremiah Horrocks Institute, University of Central Lancashire, PR1 2HE, UK; mbattarbee@uclan.ac.uk \\ ${ }^{2}$ Met Office, Exeter, EX1 3 PB, UK \\ Received 2016 December 2; revised 2017 January 13; accepted 2017 January 15; published 2017 February 14
}

\begin{abstract}
Solar energetic particles (SEPs), a major component of space weather, propagate through the interplanetary medium strongly guided by the interplanetary magnetic field (IMF). In this work, we analyze the implications that a flat Heliospheric Current Sheet (HCS) has on proton propagation from SEP release sites to the Earth. We simulate proton propagation by integrating fully 3D trajectories near an analytically defined flat current sheet, collecting comprehensive statistics into histograms, fluence maps, and virtual observer time profiles within an energy range of 1-800 MeV. We show that protons experience significant current sheet drift to distant longitudes, causing time profiles to exhibit multiple components, which are a potential source of confusing interpretations of observations. We find that variation of the current sheet thickness within a realistic parameter range has little effect on particle propagation. We show that the IMF configuration strongly affects the deceleration of protons. We show that in our model, the presence of a flat equatorial HCS in the inner heliosphere limits the crossing of protons into the opposite hemisphere.
\end{abstract}

Key words: methods: numerical - Sun: activity - Sun: heliosphere - Sun: magnetic fields - Sun: particle emission

\section{Introduction}

A significant component of space weather is the flux of solar energetic particles (SEPs), accelerated during energy release events such as flares and coronal mass ejections (CMEs) at the Sun. These high-energy charged particles can, after propagating to the Earth, disrupt satellite communications and impact astronaut health and safety (Turner 2000). Charged particles propagating through interplanetary space are guided and deflected by the solar wind's magnetic field and its spatial and temporal variations. Modern efforts in modeling space weather effects include performing numerical simulations to solve particle fluences at the Earth based on parent active region and observer locations (see, e.g., Chollet et al. 2010; Marsh et al. 2015). The most common approach is to use a transport equation (see, e.g., Roelof 1969; Aran et al. 2005; Luhmann et al. 2007), where particles are effectively bound to the interplanetary magnetic field (IMF) lines, described as the Parker spiral (Parker 1958).

Recent research (Dalla et al. 2013, 2015; Marsh et al. 2013) has shown that particle drifts, which are not modeled by a classical transport equation, play a significant role in SEP propagation to the Earth. They have been shown to be significant for protons, and especially for heavier elements (Dalla et al. 2017). Other significant factors include field-line meandering (Laitinen et al. 2016) and cross-field diffusion (Zhang et al. 2003; He et al. 2011). One significant characteristic of the IMF that has not been previously modeled in the context of SEP propagation is the heliospheric current sheet (HCS), providing the boundary between the two hemispheres of the solar dipole field. The presence of a current sheet changes the motion of charged particles by imposing two distinct regions of drifts and causing the breakdown of the guiding center motion at the sheet (Speiser 1965).

The HCS is a vast area of space where the magnetic fields associated with the northern and the southern hemispheres of the solar magnetic field transition between outward- and inwarddirected polarities. Because of the varying and complicated distribution of mean magnetic flux direction on the solar surface, and the tilt of the solar magnetic axis with respect to the rotation axis, the HCS consists of a complex 3D structure, especially at greater heliocentric distances. The HCS has been the topic of much research, but mainly from the point of view of very energetic particles called galactic cosmic rays (GCRs), propagating inward from the outer boundary of the heliosphere (references include, but are not limited to, Jokipii \& Levy 1977; Burger et al. 1985; Kóta \& Jokipii 2001; Pei et al. 2012; Strauss et al. 2012; Guo \& Florinski 2014). The role of the HCS in SEP propagation has previously been briefly investigated in Kubo et al. (2009).

In this paper, we present a first analysis of how the presence of the HCS affects the propagation of SEPs from the Sun to the Earth. We consider a flat current sheet and assess effects of current sheet thickness and different dipole configurations on SEP propagation for protons of different energies. We also present SEP time profiles at virtual observers, providing a basis of comparison with real observations.

\section{HCS Model}

In this work, we model the HCS as a flat plane separating two hemispheres of opposite polarity, with each hemisphere based on a simple, analytical magnetic field model. We model purely radial outflow of solar wind plasma, which, combined with solar rotation and flux freeze-in, results in a nonradial magnetic field. The IMF is described through spherical heliocentric coordinates as a scaled Parker spiral magnetic field $\boldsymbol{B}$

$$
\boldsymbol{B}=S(\theta) \boldsymbol{B}_{\text {Parker }},
$$

where the Parker field is defined as

$$
\begin{gathered}
B_{r, \text { Parker }}=B_{0} \frac{r_{0}^{2}}{r^{2}}, \\
B_{\theta, \text { Parker }}=0,
\end{gathered}
$$




$$
B_{\phi, \text { Parker }}=-\frac{B_{0} r_{0}^{2} \Omega_{\odot}}{u_{\mathrm{sw}}} \frac{\sin \theta}{r} .
$$

Here $B_{0}$ is the field strength at $1 r_{0}$, normalized to provide a field strength of $B(1 \mathrm{au})=3.85 \mathrm{nT}, \Omega_{\odot}=2.87 \times 10^{-6} \mathrm{rad} \mathrm{s}^{-1}$ is the average solar rotation rate, $u_{\mathrm{sw}}=500 \mathrm{~km} \mathrm{~s}^{-1}$ is the radial solar wind speed, and $S(\theta)$ is a scaling function providing the change of polarity in a gradual fashion and describing current sheet thickness. Because $S$ is only a function of colatitude $\theta$, the analytical field remains divergence-free. This simplified HCS model, where the current sheet is completely flat, is thus symmetric in respect to the heliocentric coordinate $\phi$. It is an approximation that is strictly valid only within the inner heliosphere and during solar minimum. Modeling of a nonplanar current sheet is postponed to further studies.

As the field magnitude, and thus the HCS profile, depends solely on $\theta$, thus varying along a direction perpendicular to the solar wind flow, there is no compression of the current sheet and thus no driven reconnection. Therefore, the current sheet modeled in this work does not contain additional electric fields beyond the regular motional electric field

$$
\boldsymbol{E}=-\frac{\boldsymbol{u}_{\mathrm{sw}}}{c} \times \boldsymbol{B}
$$

where $c$ is the speed of light. This electric field causes particles to experience $\boldsymbol{E} \times \boldsymbol{B}$ drift, analogous with corotation of field lines. In the case of a wavy HCS (see, e.g., Burger 2012; Pei et al. 2012; Strauss et al. 2012), especially with greater heliocentric distance, an assumed radial solar wind flow will no longer be wholly in the current sheet plane, requiring more detailed analysis of possible reconnection.

Observations estimate the HCS thickness to be in the region of between 5000 and 40,000 km at 1 au (see, e.g., Winterhalter et al. 1994; Eastwood et al. 2002). We examine the effects of a gradual transition between hemispheres and the effects of current sheet thickness. Although energetic protons may have Larmor radii much larger than the listed current sheet thicknesses, effects such as beamed injection and adiabatic focusing may cause the perpendicular velocity component of particles to be quite small, resulting in smaller than expected Larmor radii, thus warranting this approach. Thus, we define the HCS thickness shape function $S$ to be a function of colatitude through latitude $\delta=90^{\circ}-\theta$, as

$$
S(\theta)=A\left(-1+2 \mathcal{S}\left(\frac{1}{2}+\frac{2 \delta}{l_{\mathrm{HCS}}}\right)\right),
$$

where $A$ is a configuration parameter with values of +1 or -1 , $l_{\mathrm{HCS}}$ is the thickness of the HCS, and $\mathcal{S}$ is the Smootherstep function (Ebert et al. 2003), which maps the parameter range $[0,1]$ to the values $[0,1]$ as $\mathcal{S}(x)=6 x^{5}-15 x^{4}+10 x^{3}$, resulting in a smooth transition with nil first and second-order derivatives at the endpoints. Closer to the Sun, this parameterization results in smaller current sheet thicknesses. The parameter $A$ defines the polarity of the dipolar field according to cosmic-ray physics standard notation, i.e., a configuration of $A+(A=+1)$ has an outward-pointing field in the northern hemisphere, and a configuration of $A-(A=-1)$ has an inward-pointing field in the northern hemisphere, with the direction of the field in the southern hemisphere reversed. We additionally assess the validity of implementing a HCS with zero thickness, using a shape function $S_{\mathrm{H}}$, which implements the Heaviside step function $\mathrm{H}$ as

$$
S_{\mathrm{H}}(\theta)=A(-1+2 \mathrm{H}(\delta)) .
$$

Protons propagating within the fields given by Equations (1)(7) will experience drifts due to the electric field, and the gradients and curvature of the magnetic field. A full analytical treatise of particle drifts in a Parker spiral, far from the HCS, can be found in Dalla et al. (2013), where a better-suited fieldaligned frame of reference $\left(\hat{\boldsymbol{e}}_{l}, \hat{\boldsymbol{e}}_{\phi^{\prime}}, \hat{\boldsymbol{e}}_{\theta^{\prime}}\right)$ is introduced. Within this system, $\hat{\boldsymbol{e}}_{l}$ is directed along the Parker spiral magnetic field line, outward from the Sun. $\hat{\boldsymbol{e}}_{\theta^{\prime}}$ is antiparallel to the standard spherical coordinate vector $\hat{\boldsymbol{e}}_{\theta}$, and $\hat{\boldsymbol{e}}_{\phi^{\prime}}$ completes the coordinate system. Below, we summarize the nonrelativistic forms of the main drifts, the electric field, $\nabla \boldsymbol{B}$, and curvature drifts, for the simple case of a unipolar $\operatorname{IMF}(S(\theta) \equiv 1)$, as

$$
\begin{gathered}
\boldsymbol{v}_{E}=\frac{u_{\mathrm{sw}} r}{\left(r^{2}+a^{2}\right)^{1 / 2}} \hat{\boldsymbol{e}}_{\phi^{\prime}}, \\
\boldsymbol{v}_{\nabla}=\frac{\mu c}{q} \frac{r \cot \theta}{r^{2}+a^{2}} \hat{\boldsymbol{e}}_{\phi^{\prime}}-\frac{\mu c}{q} \frac{r^{2}+2 a^{2}}{\left(r^{2}+a^{2}\right)^{3 / 2}} \hat{\boldsymbol{e}}_{\theta^{\prime}}, \\
\boldsymbol{v}_{c}=-\frac{m c}{q B} v_{\|}^{2} \frac{r \cot \theta}{r^{2}+a^{2}} \hat{\boldsymbol{e}}_{\phi^{\prime}}-\frac{m c}{q B} v_{\|}^{2} \frac{r^{2}+2 a^{2}}{\left(r^{2}+a^{2}\right)^{3 / 2}} \hat{\boldsymbol{e}}_{\theta^{\prime}},
\end{gathered}
$$

where $a$ is a function of colatitude $a=u_{\mathrm{sw}}\left(\Omega_{\odot} \sin \theta\right)^{-1}$ and $\mu$ is the particle magnetic moment $\mu=m v_{\perp}^{2}(2 B)^{-1}$. Here $m$ and $q$ are the particle mass and charge, and $v_{\|}$and $v_{\perp}$ are the components of velocity parallel and perpendicular to the magnetic field, respectively. See Dalla et al. (2013) for the more general relativistic expressions.

The analytical forms show that for near-equatorial latitudes, the term aligned with $\hat{\boldsymbol{e}}_{\theta^{\prime}}$ dominates both curvature and gradient drifts. For both these drifts, when considering the two polarity configurations of the solar dipolar field, we find that for $A+$, both hemispheres cause drift of positively charged particles toward the equator, and for $A-$, away from it, which are the patterns well known from GCR studies. Thus, for the $A+$ configuration, the equator is a stable position, and for the $A-$ configuration, a labile position.

Inclusion of the HCS, for example, defined through a shape function $S(\theta)$, will cause additional drifts due to a change of the magnetic field as a function of $\theta$. The first drift, valid for both smooth and step-mode current sheet profiles, is the current sheet drift, described commonly as Speiser motion (Speiser 1965). With $B$ approaching zero, the guiding center approximation of particle motion breaks down. Particles oscillate between the two magnetic field polarities by performing partial gyromotion in each side, then crossing the sheet, and performing gyromotion of opposite chirality on the other side. For particles with positive charge, this motion is in a western direction for $A+$ and an eastern direction for $A-$. For a step-mode field transition and an isotropic distribution, this was found to lead to an average velocity of $\left\langle v_{\mathrm{S}}\right\rangle=0.463 v$ (Burger et al. 1985).

If the gyroradius of particles is smaller than the characteristic length scale describing the rate of change for $\boldsymbol{B}$ due to the shape function $S(\theta)$, a second drift is found at the current sheet, 
taking the form of classical gradient drift, and defined as

$$
\boldsymbol{v}_{\mathrm{g}}=\frac{c m}{2 q} \frac{v_{\perp}^{2}}{B^{3}} \boldsymbol{B} \times(\nabla B)
$$

If this drift is present, then $\nabla B$ would be aligned with $\theta^{\prime}$, leading to the gradient drift being aligned with $\phi^{\prime}$. The direction of this gradient drift would be opposite to that of current sheet drift (or Speiser motion).

\section{Simulations}

In our simulations, we investigate SEP trajectories in the fixed frame (spacecraft frame) in the presence of a flat HCS using a numerical test-particle model (Dalla \& Browning 2005; Kelly et al. 2012) with modifications suited to heliospheric problems introduced in Marsh et al. (2013). Instead of using the focused transport equation (see, e.g., Roelof 1969), we solve the full 3D differential equations of motion for each particle. In our model, drifts are not introduced into the relevant equations analytically, but instead arise naturally from the Lorentz and electric forces generated by the fields given by Equations (1)(7) acting on particles during each step of their motion.

We simulate the propagation of energetic protons, injected instantaneously at time $t=0$ from a heliocentric distance of $2 R_{\odot}$. Protons are injected from a region with an angular extent of $6^{\circ} \times 6^{\circ}$, centered at the heliographic equator. For select studies, the injection latitude was varied in order to assess latitudinal drifts. Particles have initial pitch angles pointing in a random direction within a hemisphere directed outward from the Sun along the Parker spiral. The relativistic differential equations of particle motion and acceleration are solved using a self-optimizing Bulirsch-Stoer method (Press 1996). Particles are propagated in the prescribed magnetic and electric fields, where the motional electric field is solved using a solar wind speed of $u_{\mathrm{sw}}=500 \mathrm{~km} \mathrm{~s}^{-1}$. In order to model the effects of turbulence and wave-particle effects, particles experience large-angle scattering in the solar wind frame, with Poisson-distributed scattering intervals, and a constant rigidityindependent mean free path of $1 \mathrm{au}$, in agreement with an assumed low level of scattering.

We inject $N=10^{5}$ particles and trace their propagation within the heliosphere for $100 \mathrm{hr}$. Snapshots of particle profiles are provided every 60 minutes. A collection sphere is placed at a heliocentric distance of $1 \mathrm{au}$, over which crossings are tracked, allowing the generation of fluence maps, histograms, and virtual observer time profiles. Fluence maps were generated with tiles of an angular extent of $1^{\circ} \times 1^{\circ}$ over the full length of the simulation, whereas time profile generation used $6^{\circ} \times 6^{\circ}$ windows and 30-minute time binning.

We chose eight different magnetic field configurations for use in our simulations. As reference cases, we simulated particle propagation in both inward- and outward-pointing unipolar fields $(S(\theta)= \pm 1)$. For HCSs we used three different thicknesses by varying the parameter $l_{\mathrm{HCS}}$ in Equation (6). Each current sheet thickness was simulated for both $A+$ and $A$ - configurations, as described in Section 2. The current sheet was simulated with thicknesses of 0,5000 , and $40,000 \mathrm{~km}$ at 1 au. The first case was in fact modeled as a Heaviside step function using the shape function (7). A plot of $S(\theta)$ at 1 au for various current sheet thicknesses is shown in Figure 1. The shown thicknesses of $5000 \mathrm{~km}$ and $40,000 \mathrm{~km}$ at 1 au
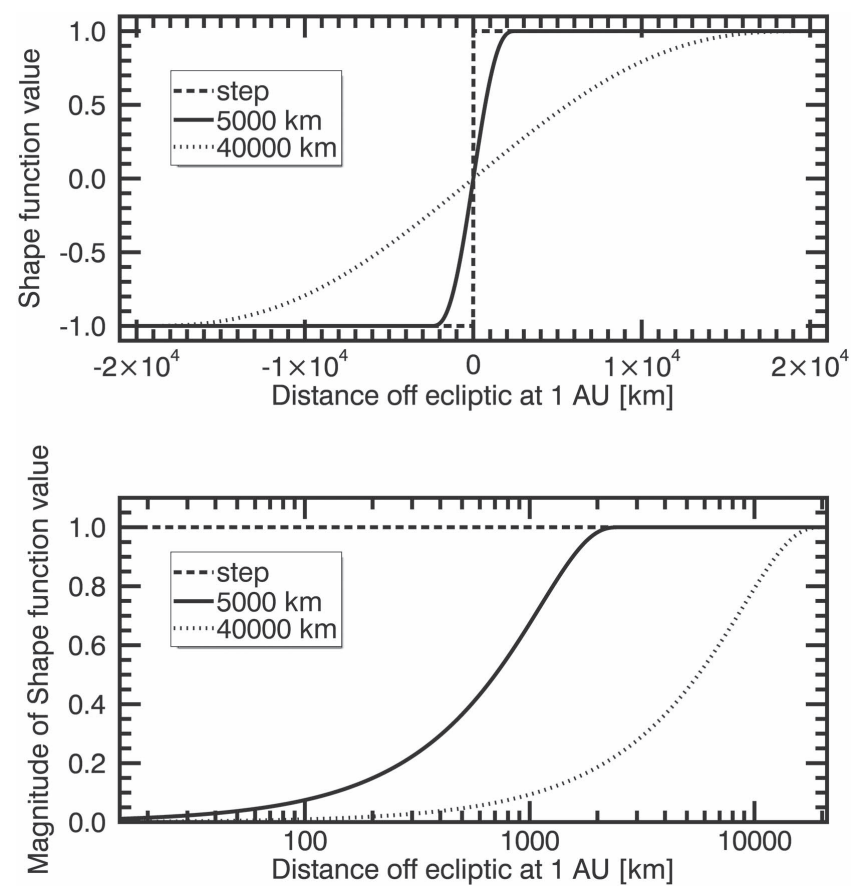

Figure 1. Shape function $S(\theta)$ as seen at a heliocentric distance of $1 \mathrm{au}$. The dashed, solid, and dotted lines correspond with HCS thicknesses of $0 \mathrm{~km}$, $5000 \mathrm{~km}$, and $40,000 \mathrm{~km}$, respectively. The shape function is displayed with both linear (top) and logarithmic (bottom) distance from the heliographic equator, where the logarithmic plot shows only the positive half of the function.

correspond with angular extents of 0.0019 and 0.015 , respectively.

In order to simulate the infinitesimally thin current sheet, henceforth referred to as the Heaviside case, we could not use the regular Bulirsch-Stoer algorithm, as it could not automatically optimize particle propagation over the step function. Instead, we used an adaptive-step leapfrog Boris-push method (Boris 1970), which is a solver commonly used in Particle-InCell codes.

For each run, protons are injected as either monoenergetic populations with initial energies of $1,10,40,100,400$, or $800 \mathrm{MeV}$, or as a power law between 10 and $400 \mathrm{MeV}$ with a spectral index of $\gamma=-1.1$.

\section{Results}

Our first step was to perform a qualitative assessment of apparent HCS drifts as a function of sheet thickness. In Figure 2 we show comparisons between all eight simulated IMF configurations. We plot the distribution of protons injected at $100 \mathrm{MeV}$ after $1 \mathrm{hr}$ of propagation flattened to the $x-y$ plane (the equatorial plane of the Sun, with the $x$-axis pointing in the direction of $0^{\circ}$ longitude). Injection was centered at $\left(0^{\circ}, 0^{\circ}\right)$. The unipolar cases (leftmost column) show that within $1 \mathrm{hr}$, little drift has taken place. The three $A+$ panels (top row) show that the presence of a current sheet generates significant current sheet drift to the right (west), and the three $A$ - panels (bottom row) show current sheet drift to the left (east). Gradient drift associated with the variation of $B$ over the thickness of the current sheet is found to be negligible.

We also performed a check to verify that the protons that appear to have drifted are indeed drifting protons, not a projection effect due to the $x-y$ plot. Protons experiencing current sheet drift were confirmed to be located in the vicinity 

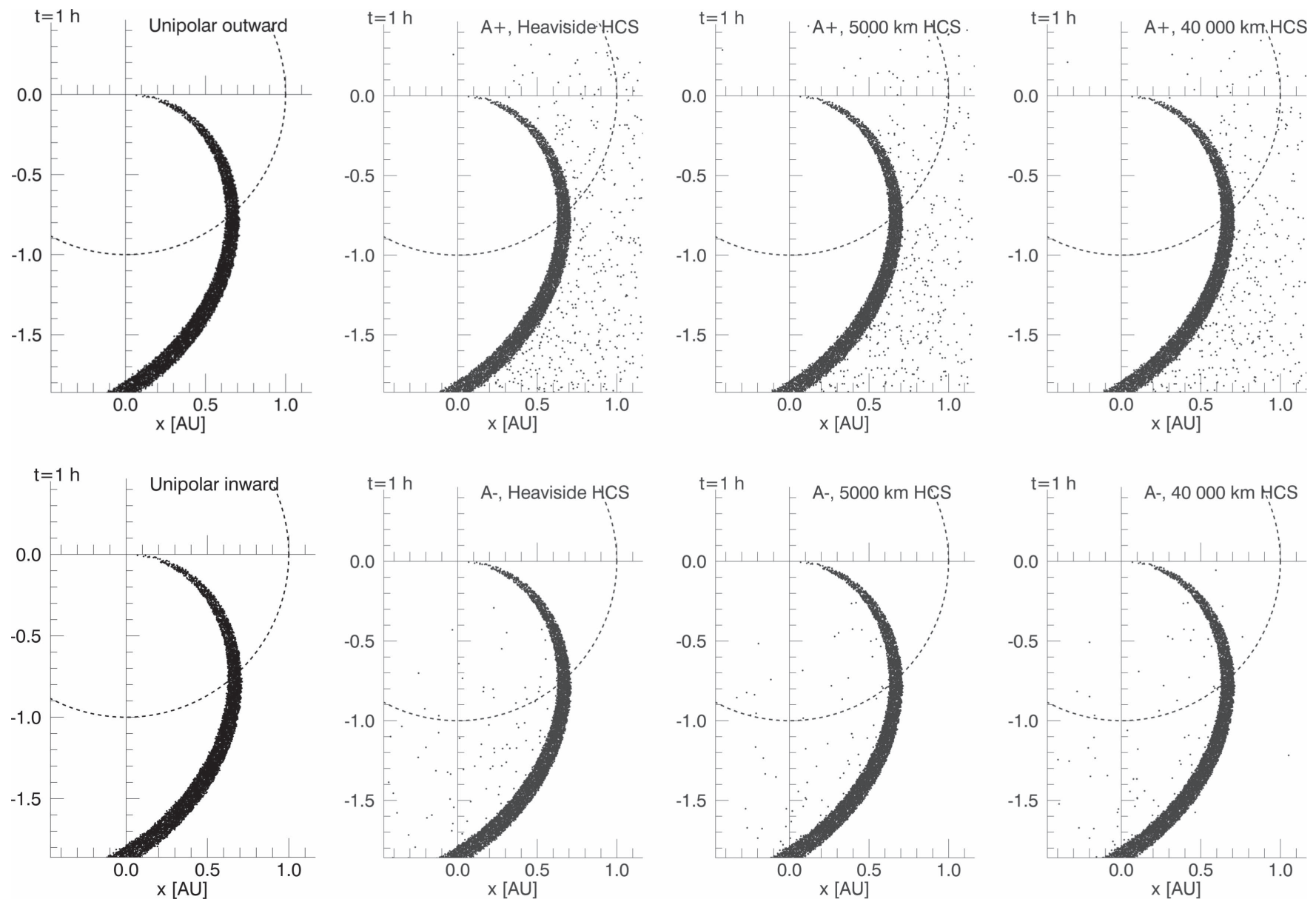

Figure 2. Projections of protons injected at $100 \mathrm{MeV}$, after $1 \mathrm{hr}$ of simulation, onto the $x-y$ plane for eight different magnetic field configurations. Top row, from left: outward-pointing unipolar field, followed by $A+$ configurations with current sheet thickness parameters corresponding with 1 au thicknesses of $0 \mathrm{~km}$ (Heaviside step), $5000 \mathrm{~km}$, and 40,000 km. Bottom row, from left: inward-pointing unipolar field, followed by $A$ - configurations with current sheet thickness parameters corresponding with 1 au thicknesses of $0 \mathrm{~km}$ (Heaviside step), $5000 \mathrm{~km}$, and 40,000 km. A distance of 1 au is displayed with a dashed circle. The proton spreads show that current sheet drift (top row: to the right, bottom row: to the left) is noticeable for all current sheets.
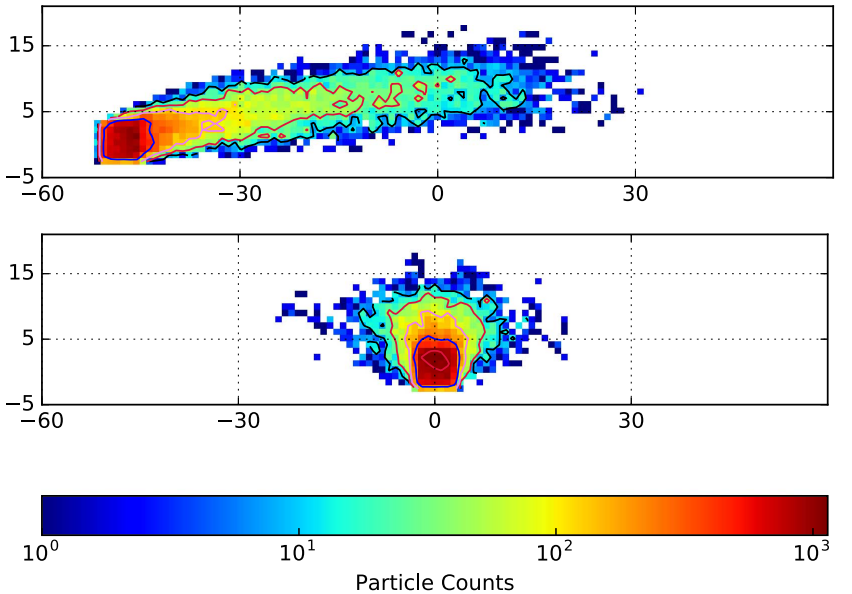

Figure 3. Maps of protons, injected at $100 \mathrm{MeV}$, crossing the 1 au sphere using $1^{\circ} \times 1^{\circ}$ binning for a unipolar inward-pointing magnetic field. Fluence colors and contours are on a logarithmic scale, with two contours per decade. Top panel: proton crossing coordinates (in degrees) relative to injection site, showing how the $\boldsymbol{E} \times \boldsymbol{B}$ drift and latitudinal drifts both work concurrently. Bottom panel: proton crossing coordinates relative to the best-connected field line with the effects of $\boldsymbol{E} \times \boldsymbol{B}$ drift removed.

of the HCS. Plots performed for other proton energies show comparable results, with an increase in proton energy resulting in greater deviation from the well-connected field lines. At later stages of the simulation, up to $100 \mathrm{hr}$, the distribution of protons in the inner heliosphere remains characteristically comparable with the $1 \mathrm{hr}$ case, although corotation causes a westward transition of all protons, and the general propagation of protons outward from the Sun causes the proton counts close to the Sun to decrease.

In order to assess the magnitude of proton drifts, we gathered all proton crossings across the 1 au sphere, saving the time of crossing, the longitude, and the latitude of each proton. In Figure 3 we show a map of $100 \mathrm{MeV}$ proton crossing counts, for a unipolar inward-pointing magnetic field, relative to the injection coordinates, using $1^{\circ} \times 1^{\circ}$ binning, adding up all counts over the $100 \mathrm{hr}$ duration of the simulation (top panel). We also show a comparative picture where we have removed the effects of corotation (bottom panel). Corotation, also described as the $\boldsymbol{E} \times \boldsymbol{B}$ drift, is caused by the field lines along which the particles propagate being frozen into the radially outflowing solar wind plasma, resulting in the intersection points at 1 au being rotated westward. We also added a longitudinal offset to the bottom panel, so coordinates are shown in relation to the best-connected field line. Henceforth, we will utilize these corrections.

The proton distributions in Figure 3 show that the effect of corotation is significant, which is unsurprising considering the $100 \mathrm{hr}$ extent of the simulation. The strongest fluence is found 

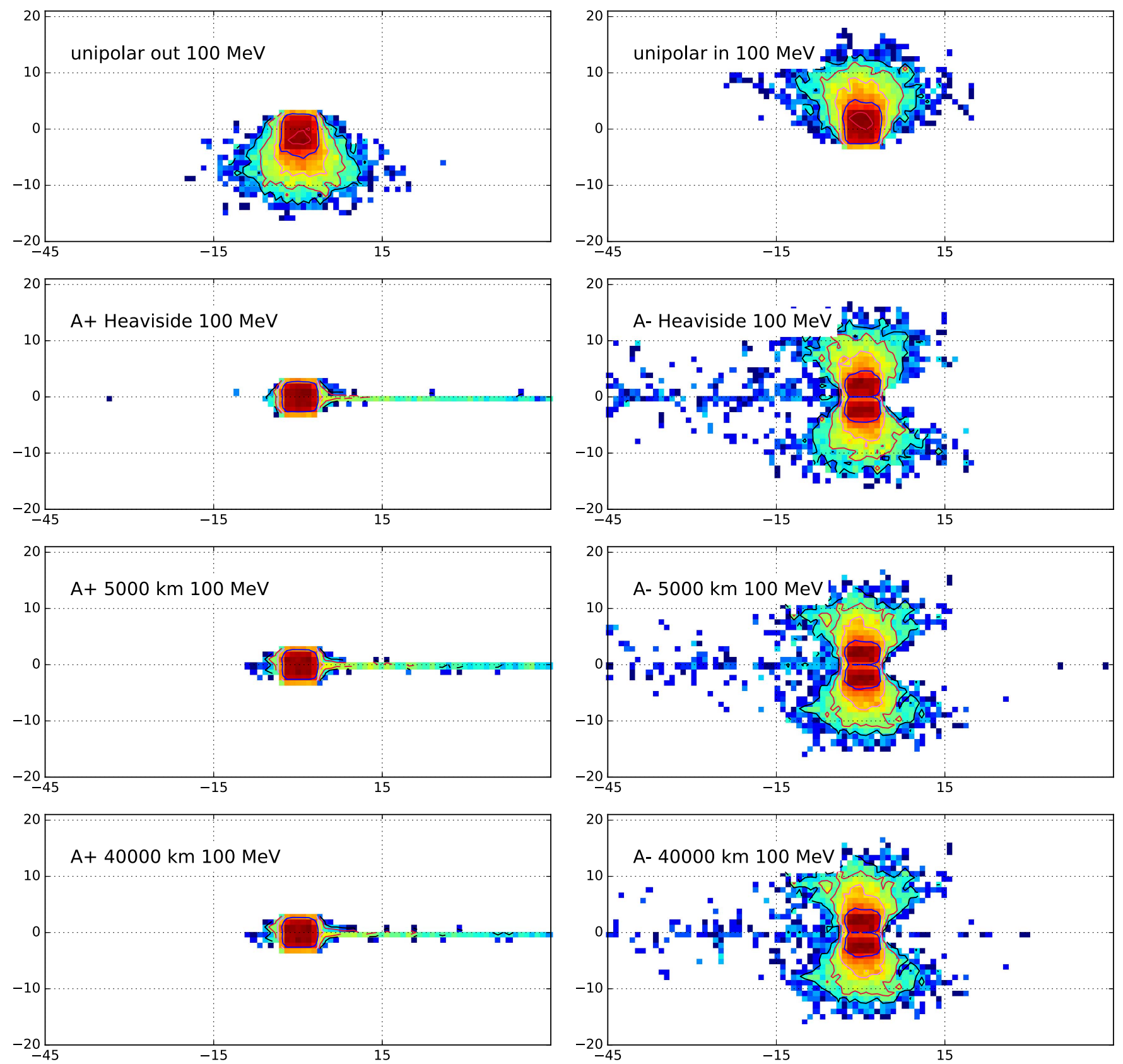

Figure 4. Maps of crossings of protons, injected at $100 \mathrm{MeV}$, across the 1 au sphere, over a time of $100 \mathrm{hr}$, relative to the best-connected field line at injection time, with the effects of corotation removed. Fluence colors and contours are on a logarithmic scale, with two contours per decade. Top row: unipolar field, pointing outward (left) and inward (right). Second to fourth rows: HCS thickness scaled to $0 \mathrm{~km}, 5000 \mathrm{~km}$, and 40,000 km at 1 au, respectively, with an $A+$ (left column) or $A$ - (right column) field configuration.

at the well-connected field line. A drift in latitude (upward for this polarity) is seen, as well as one in longitude, moving protons away from the well-connected field lines (see also Marsh et al. 2013).

We now refer to Dalla et al. (2013) as a theoretical basis of drift analysis. The strongest drifts in longitude (gradient and curvature) are found to be proportional to a function $g(\theta)$, which approaches zero at the equator. This explains protons displaying significant longitudinal drift only after having drifted to higher latitudes. In this field configuration, gradient drift $\left(\propto v_{\perp}^{2}\right)$ pushes protons to the west, whereas curvature drift $\left(\propto v_{\|}^{2}\right)$ causes drift toward the east. Both gradient and curvature drifts are in the same latitudinal direction, which for this field configuration is to higher colatitudes. The polarization drift is of smaller magnitude, and thus, ignored in this work.

In the presence of the HCS, the latitudinal drifts in each hemisphere play a significant role to how protons propagate (see, e.g., Jokipii \& Levy 1977). For protons injected at and near the HCS, as in our simulations, we find the dynamics presented to differ significantly from the unipolar case. In Figure 4, we plot fluence maps of 1 au crossings of protons, injected at $100 \mathrm{MeV}$, for all eight simulated IMF configurations, in the same format as in the lower panel of Figure 3. The latitudinal drifts in the $A+$ configuration are found to efficiently trap protons close to the current sheet, where they experience current sheet drift. For the $A-$ configuration, curvature and 

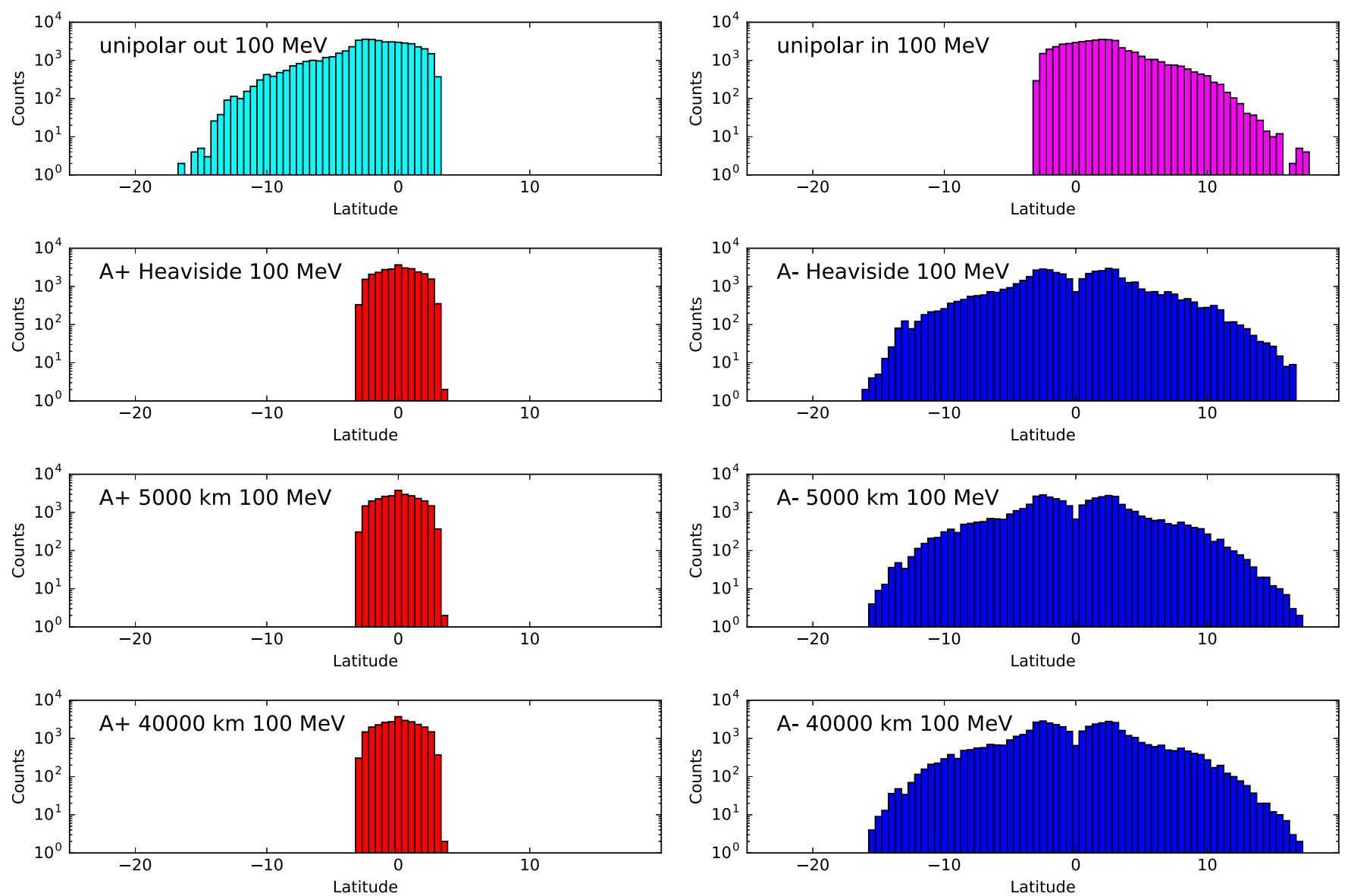

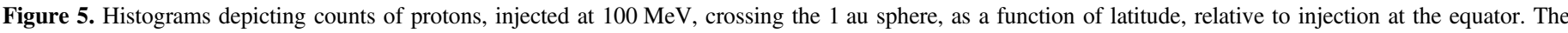
layout is as in Figure 4.

gradient drifts push protons away from the HCS, but Speiser motion nevertheless allows some protons to propagate along the current sheet until they are ejected and drift away from it. As our model does not include an intrinsic electric field at the sheet, ejection happens because of particle scattering.

Protons experiencing current sheet drift are visible at western heliolongitudes for the $A+$ configuration (left column) and at eastern heliolongitudes for the $A-$ configuration (right column). Of particular note for the $A-$ configuration is how, if looking closely at the cells closest to the current sheet at the best-connected field line, intensities are smaller than just above or below it. In Figure 5, we plot histograms of $100 \mathrm{MeV}$ proton latitudes at the time they cross the 1 au sphere boundary, for eight different IMF configurations. For an $A+$ configuration, protons are preferentially located at the center of the current sheet, whereas for the $A$ - configuration, two peaks farther out are seen. Thus, the depletion at the sheet is shown to be real, not caused by current sheet drift spreading a constant amount of protons over a wider range of longitudes.

In Figure 6, we plot histograms of $100 \mathrm{MeV}$ proton longitudes at the time they cross the 1 au sphere boundary, for eight different IMF configurations. The current sheet drift is seen to have a significant effect, allowing protons to wrap at least $180^{\circ}$ around the Sun. An $A+$ configuration is seen to have a slightly stronger current sheet drift, which is in agreement with the equator being a stable position in $A+$, and a labile position in $A-$.
In Figure 7, we display histograms of proton longitudes at the time they cross the 1 au sphere boundary, for energies of $10,40,100$, and $400 \mathrm{MeV}$. The left column shows results for an IMF with an $A+$ configuration, the right column for one with an $A$ - configuration, with HCS thickness set to $5000 \mathrm{~km}$ at $1 \mathrm{au}$. Both the maximum amount drifted and the count of protons at each drifting distance are found to increase with energy. This is as expected, as faster protons are able to sweep across the current sheet from a wider region due to a larger gyroradius, and also due to average Speiser motion being linked with energy (Burger et al. 1985). The $A-$ configuration displays a much stronger energy dependence for current sheet drift, as the reach of particle gyromotion plays a critical role in sampling of the magnetic field reversal, due to lateral drifts transporting protons away from the HCS. The main peak for $A-$, however, does spread out, as longitudinal drifts outside the current sheet can also cause protons to spread westward.

The maps and histograms presented in Figures 3 through 7 do not explicitly display the time profiles of proton crossings at 1 au. In order to allow comparisons with real-world observations, we simulated virtual observers at $1 \mathrm{au}$, by collecting proton counts over neighboring regions of $6^{\circ} \times 6^{\circ}$ extent on the surface of the 1 au sphere. For this analysis, we performed simulations using a proton injection distribution given by a power law with $\gamma=-1.1$, extending from $10 \mathrm{MeV}$ to $400 \mathrm{MeV}$. We inject $N=10^{6}$ particles in order to attain better statistics. For gathering of time profiles, we introduced 

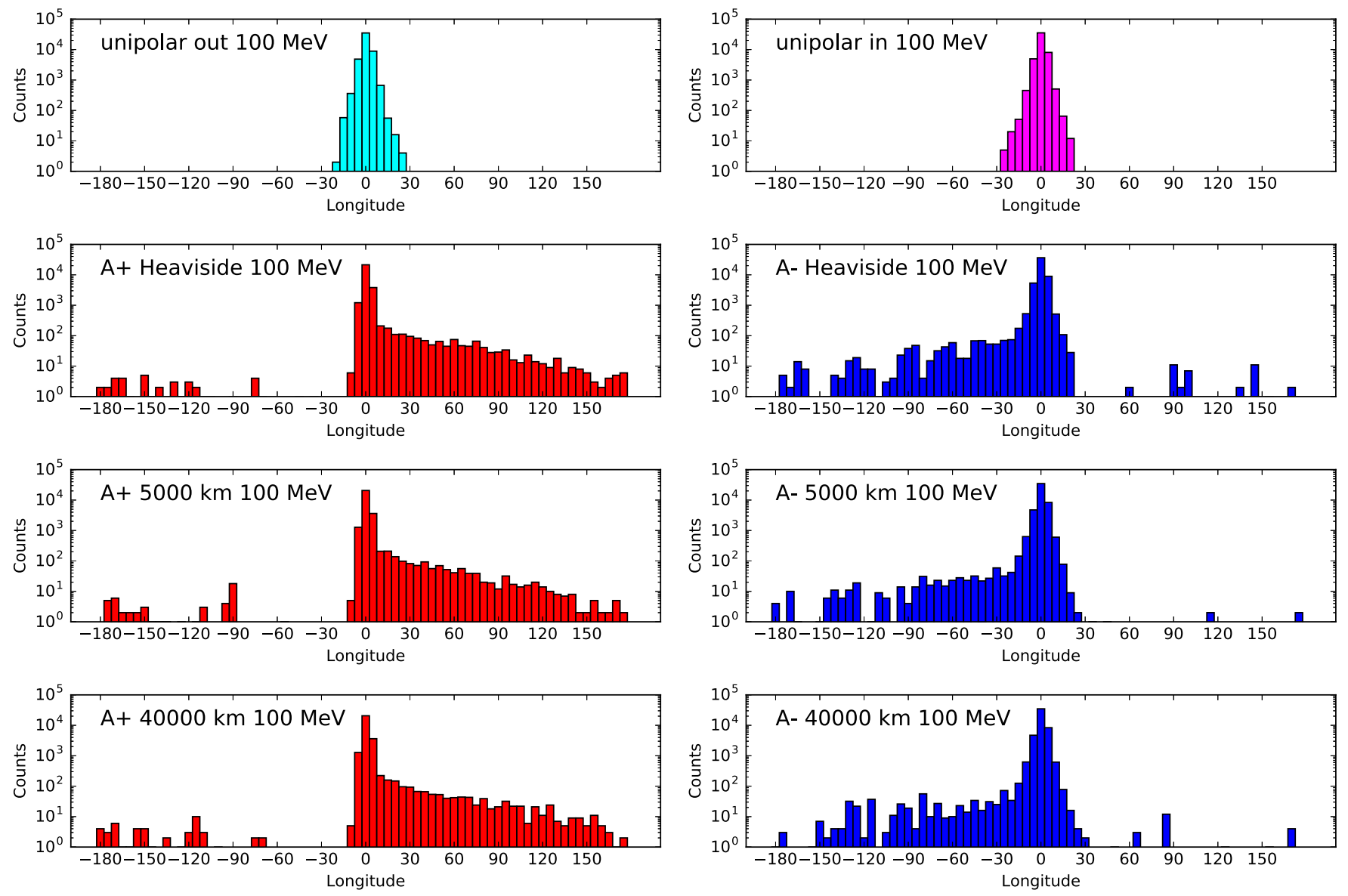

Figure 6. Histograms depicting counts of protons, injected at $100 \mathrm{MeV}$, crossing the 1 au sphere, as a function of longitude, relative to the best-connected field line. The effect of corotation has been removed. The layout is as in Figure 4.

energy channels spanning the extents of $10.0-40.0 \mathrm{MeV}$, 60.0-100.0 MeV, and 200.0-400.0 MeV. In Figures 8-10, we display time profiles for an outward-pointing unipolar field, an $A-$ IMF configuration, and an $A+$ IMF configuration, respectively.

The unipolar field depicted in Figure 8 shows how a single injection event can cause different kinds of observations, depending on virtual observer location, similar to the findings of Marsh et al. (2015). At the best-connected field line, the proton flux increases abruptly and then decays exponentially. At eastern longitudes, flux is nearly nonexistent. With increasing longitudinal separation to the west, the onset is delayed and the shape of the profile becomes more gradual. As flux at western longitudes is influenced by corotation, high-energy protons are less numerous, because they have propagated rapidly out of the inner heliosphere. At negative heliolatitudes, where in our setup all counts are due to drifting effects, we also find an abrupt rise in flux at connected longitudes and slower rises at western longitudes. However, because latitudinal drifts are energy dependent, these time profiles emphasize high-energy protons. Thus, if the proton flux of a solar event at an observer is strongly influenced by latitudinal drifts, the observed spectrum can appear much harder than that of the source population. We also note that separation between the observer and the well-connected field line increases the onset time difference between different energies.

With the $A-$ IMF configuration, shown in Figure 9, we see a case very similar to the unipolar one, with rapid or prolonged rise phases of intensity, depending on longitude. For this case, however, intensities extend to both positive and negative heliolatitudes. Again, the process of latitudinal proton drifts causes apparent hardening of observed proton spectra north and south of the injection region. We also note that a relatively small abrupt component is seen at the equator, at eastern longitudes, because of protons experiencing current sheet drift. Owing to the combined effect of current sheet drift and latitudinal drifts, high-energy protons are much less abundant at western longitudes than for the unipolar case.

With the $A+$ IMF configuration, shown in Figure 10, we find that the gradient and curvature drifts prevent any significant proton flux from extending to positive or negative heliolatitudes. Protons at lower energies display the same longitudinal characteristic of more prolonged event rise with increasing longitude. Of particular interest is the abrupt current sheet drift associated component at early phases of the simulation, as can be seen by comparing Figure 10 with Figure 8 . This additional component is found for western observers, causing the time profiles to exhibit two distinct components. Thus, a single injection event could, with a suitable IMF configuration, be observed as two particle events.

Comparisons of different HCS thickness parameters did not result in noticeable variation in the characteristics of proton time profiles. The additional plots have thus been omitted.

Solar active regions are usually associated with sunspots above or below the solar equator. The results presented in Figures $8-10$ are applicable if the acceleration region, for 

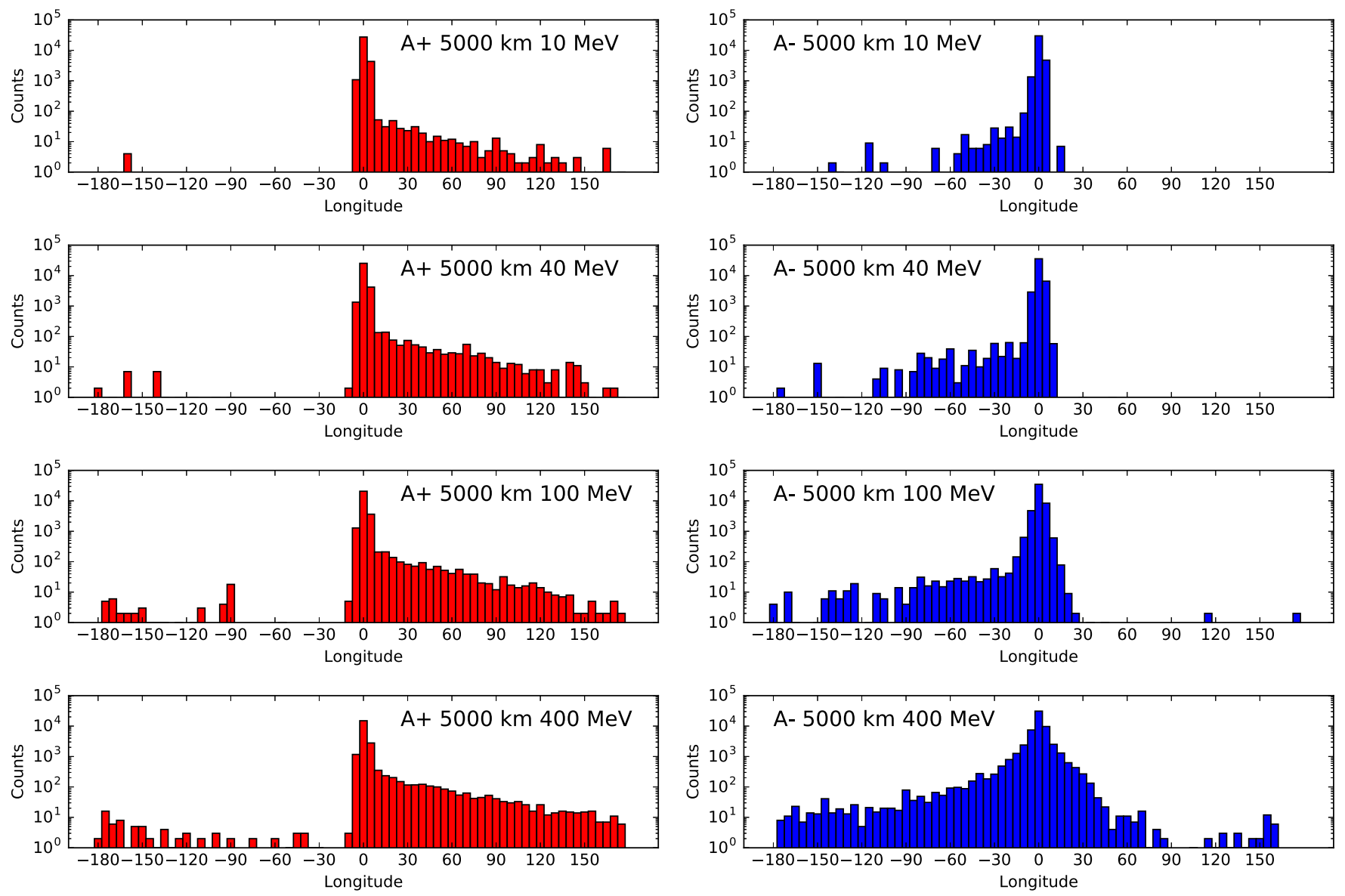

Figure 7. Histograms depicting counts of protons, crossing the 1 au sphere, as a function of longitude, relative to the best-connected field line. The effect of corotation has been removed. The left column depicts results for an IMF with an $A+$ configuration, the right column for $A-$. HCS thickness was scaled to $5000 \mathrm{~km}$ at 1 au. From the top to the bottom rows, histograms are shown for injection energies of $10,40,100$, and $400 \mathrm{MeV}$. At high energies, the current sheet drift of protons is seen to wrap around past $180^{\circ}$.

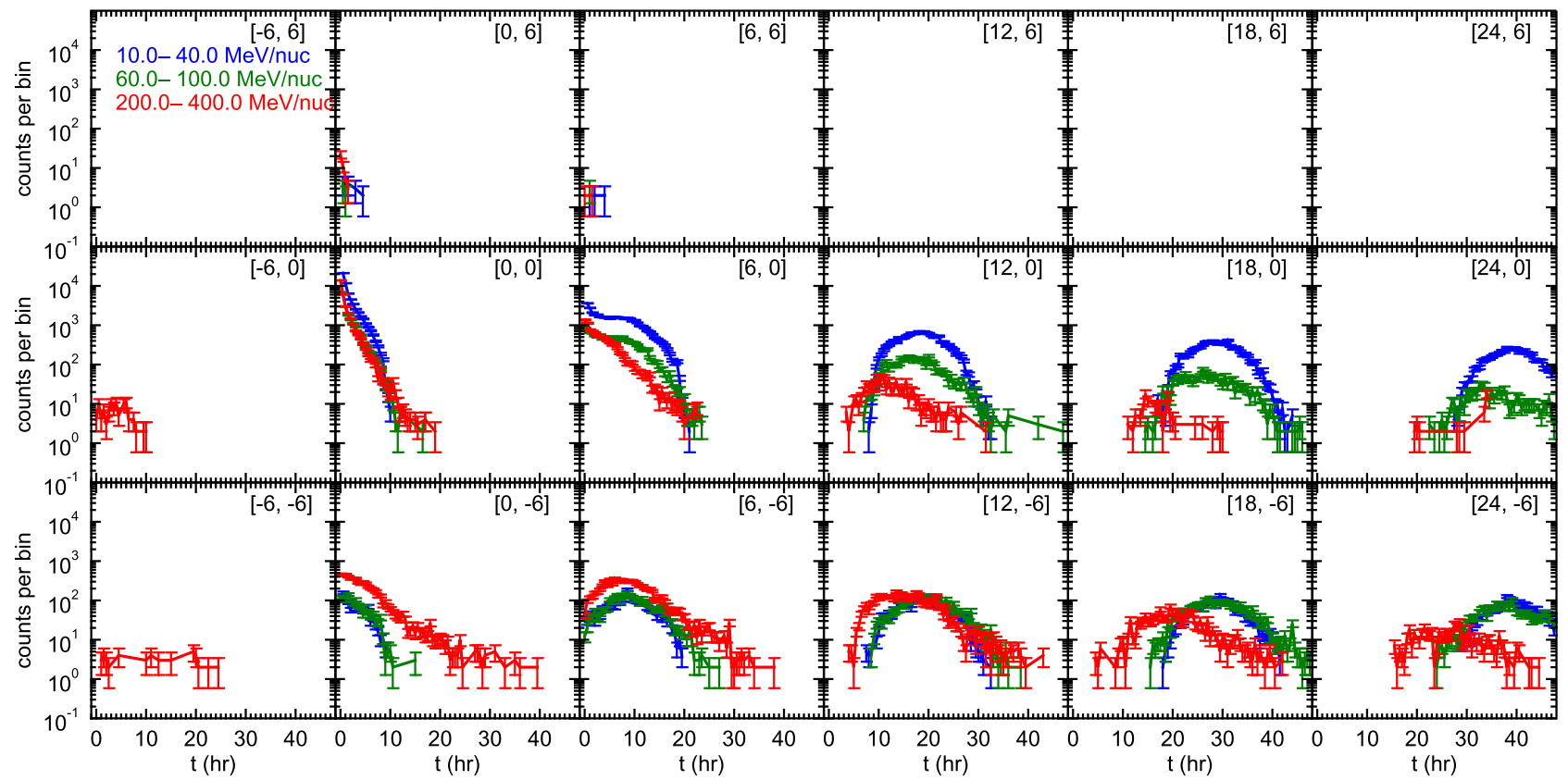

Figure 8. Time profiles of protons, where the IMF is a unipolar outward-pointing field. Each panel shows profiles for $4 \pi$ steradian and $6^{\circ} \times 6^{\circ}$ angular extent virtual observers at 1 au, with the captions indicating the (longitude, latitude) offset in degrees from the position of the best-connected field line. Time profiles were generated over an extent of $48 \mathrm{hr}$, with 30-minute time binning. Injection was at the heliographic equator, with a power law of $\gamma=-1.1$ and an injection energy range spanning $10-400 \mathrm{MeV}$. Curvature and gradient drifts cause virtual observers at lower latitudes to see some counts. 


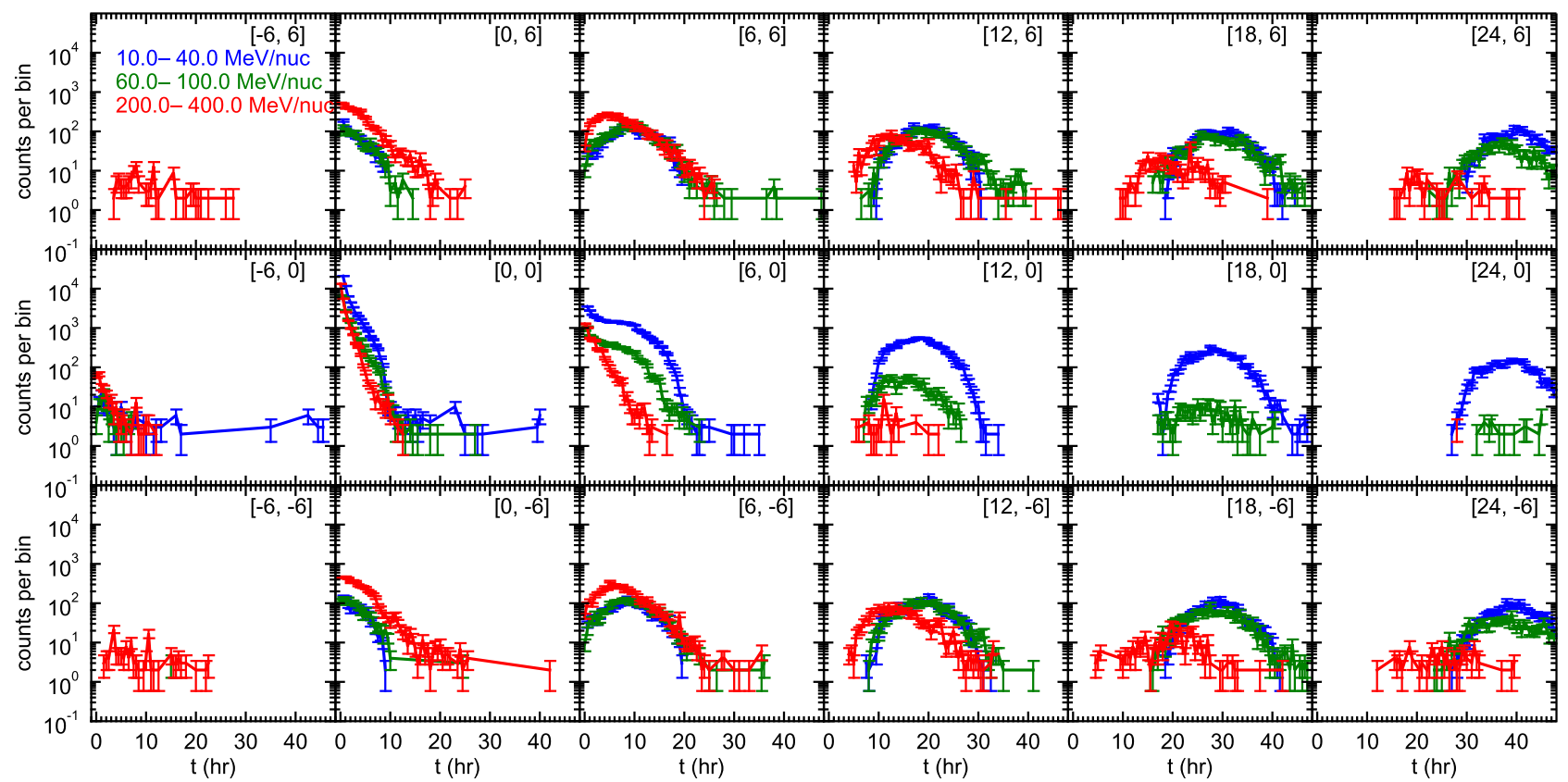

Figure 9. Time profiles of protons, where the IMF has an $A$ - configuration, with HCS thickness scaled to $5000 \mathrm{~km}$ at 1 au. Other properties are as in Figure 8 . Curvature and gradient drifts cause virtual observers at both lower and higher latitudes to also see some flux.

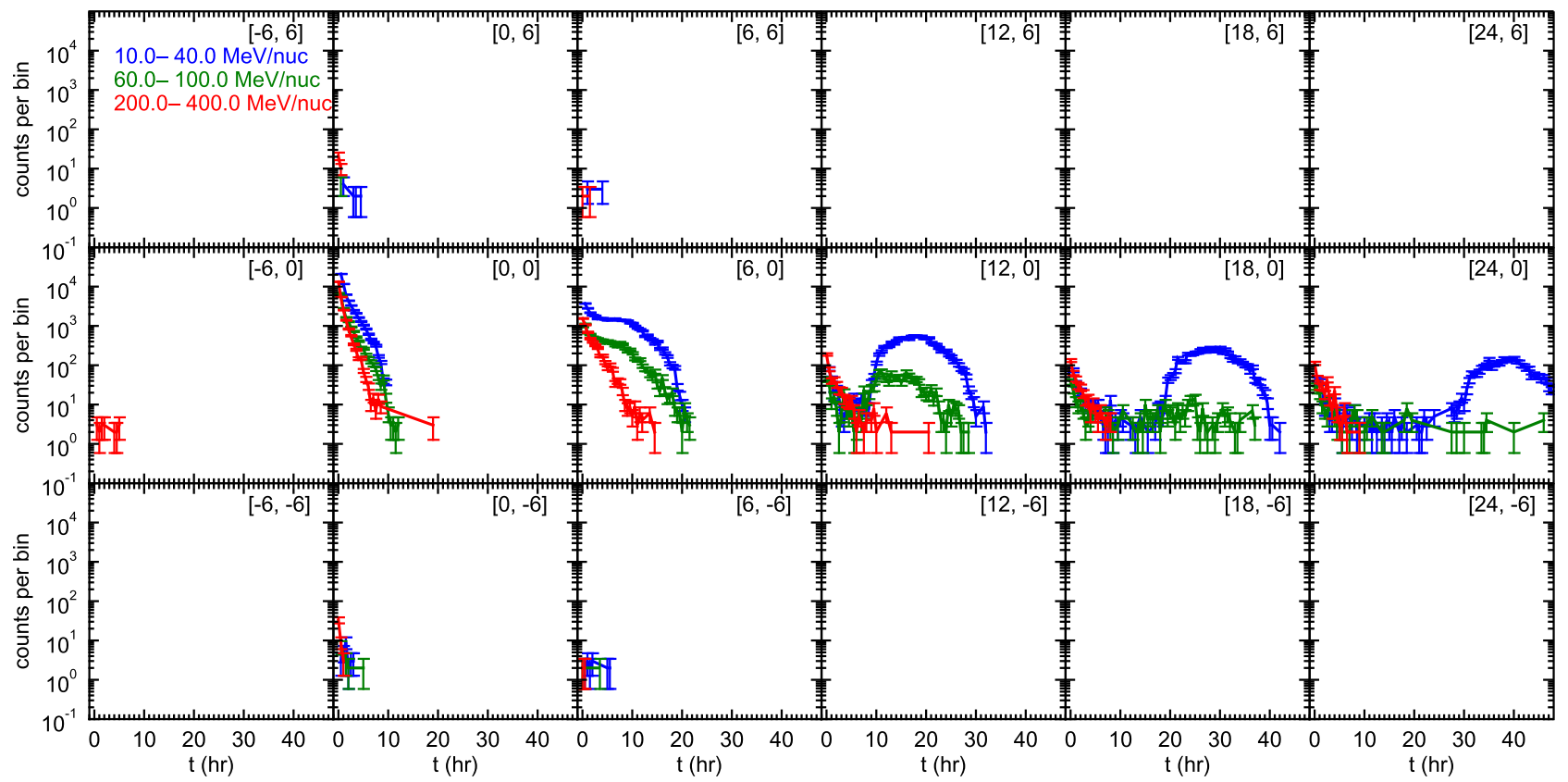

Figure 10. Time profiles of protons, where the IMF has an $A+$ configuration, with HCS thickness scaled to $5000 \mathrm{~km}$ at 1 au. Other properties are as in Figure 8 . Curvature and gradient drifts constrain proton flux to the best-connected latitude. Of particular note is the two-component time profile seen at western locations, with an impulsive peak close to injection time in addition to the delayed gradual increase seen with other IMF configurations.

example, a coronal shock front, spans all the way to the equator. If the injection location is above the HCS, it will take time for particles to reach it and feel its effects. In Figure 11, we display virtual time profiles for an observer at the heliographic equator, when the injection region of $6^{\circ} \times 6^{\circ}$ was centered at a latitude of $+6^{\circ}$. The top row shows profiles for a unipolar outward-pointing IMF, and the bottom row for an IMF with an $A+$ HCS configuration. For high-energy protons, the HCS facilitates arrival at the observer location earlier than for a unipolar field. However, as the HCS spreads protons across a wide range of longitudes, the achieved peak flux is lower than that without the HCS. At low energies the difference between the two cases is insignificant, possibly because of protons drifting close to the equator but not quite reaching the current sheet.

In Figure 12 we show fluence maps for the same simulations as seen in Figures 8 through 10. Drifts extend protons for significant distances in latitude and longitude. The relative spread, compared with Figure 4, is not drastically different, as the number of injected particles for the power-law runs was increased tenfold. One should note that contours are spaced two per decade. 

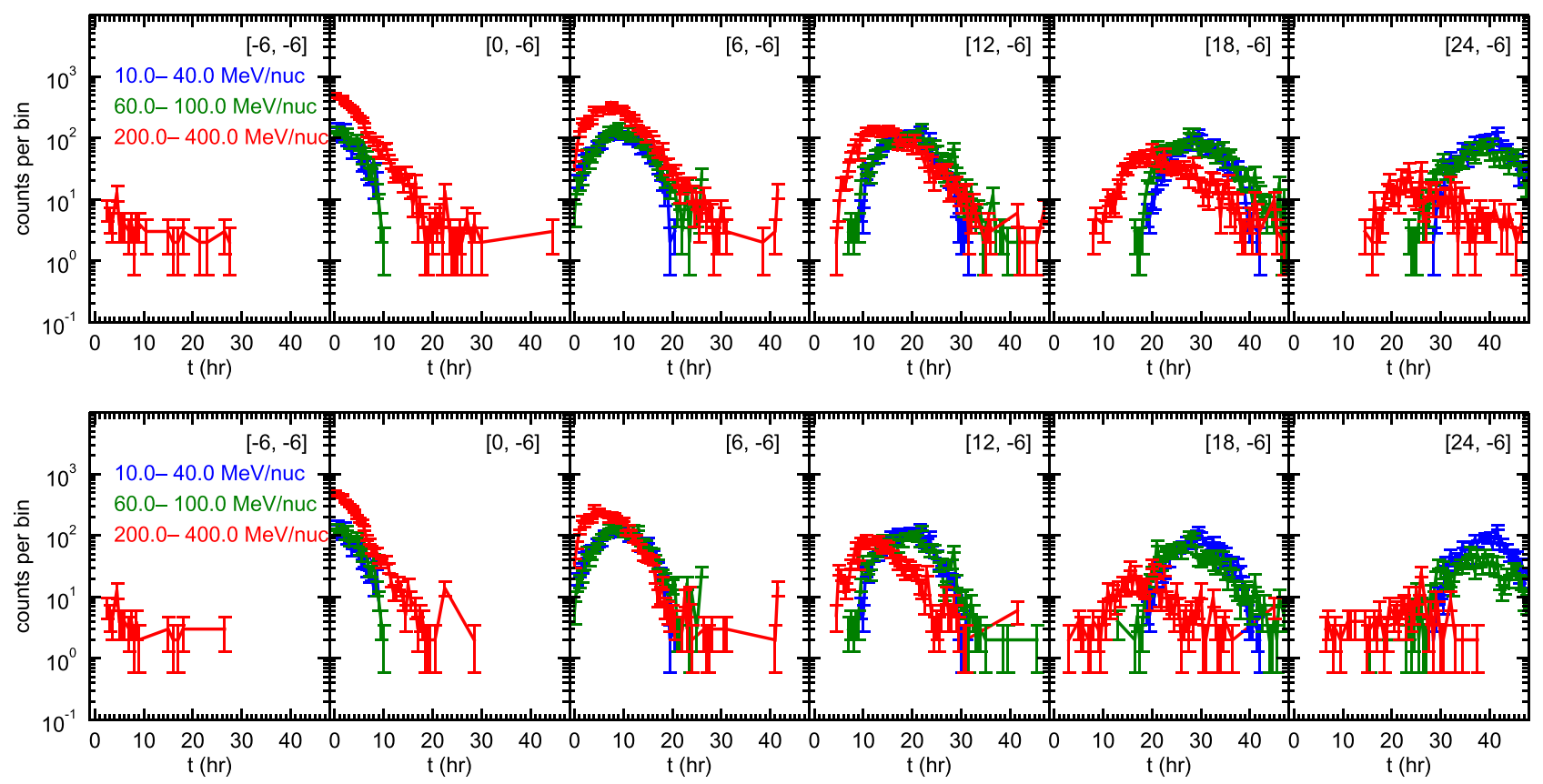

Figure 11. Time profiles of protons, where the IMF is a unipolar outward-pointing field (top row) or where the IMF has an $A+$ configuration with HCS thickness scaled to $5000 \mathrm{~km}$ at 1 au (bottom row). Each panel shows profiles for $4 \pi$ steradian and $6^{\circ} \times 6^{\circ}$ angular extent virtual observers at 1 au, with the captions indicating the (longitude, latitude) offset in degrees from the position of the best-connected field line. Injection was at a heliographic latitude of $+6^{\circ}$, thus all observers are centered on the solar equatorial plane. Injection was with a power law of $\gamma=-1.1$ and an injection energy range spanning $10-400 \mathrm{MeV}$. Time profiles were generated over an extent of $48 \mathrm{hr}$, with 30-minute time binning.
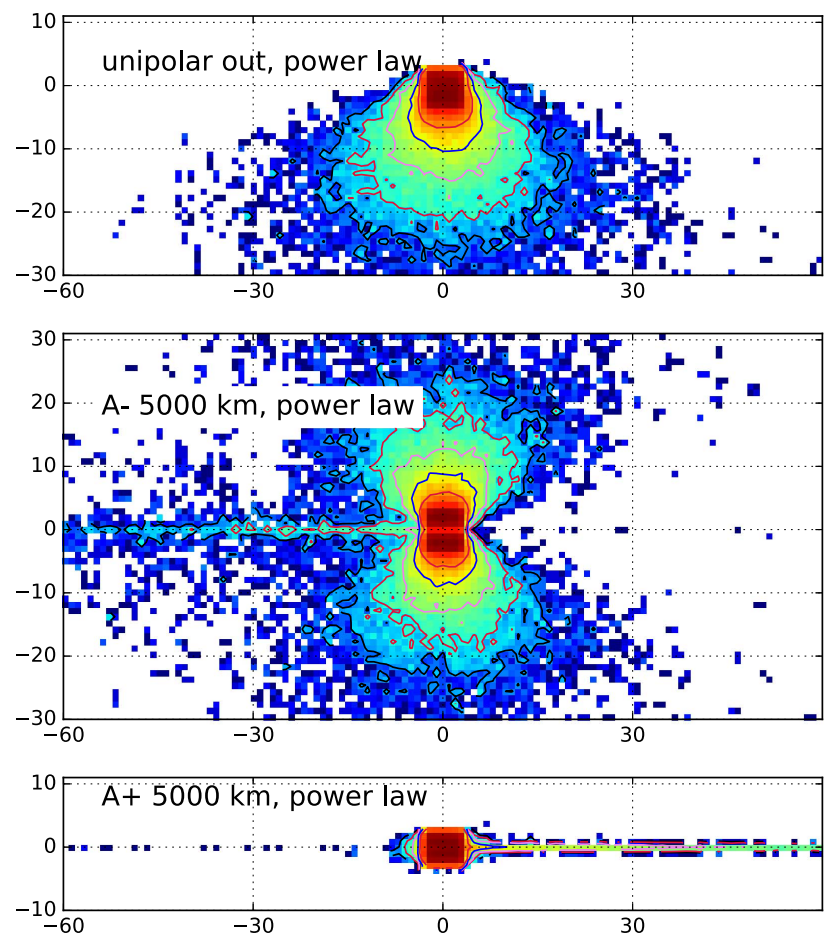

Figure 12. Maps of crossings of protons, injected from a power law with $\gamma=-1.1$, spanning the energy range from 10 to $400 \mathrm{MeV}$, across the 1 au sphere, over a time of $100 \mathrm{hr}$, relative to best-connected field line, with the effects of corotation removed. Fluence colors are on a logarithmic scale, with two contours per decade. Top: unipolar field, pointing outward. Middle: HCS thickness scaled to $5000 \mathrm{~km}$ at $1 \mathrm{au}$, with an $A$ - field configuration. Bottom: HCS thickness scaled to $5000 \mathrm{~km}$ at 1 au, with an $A+$ field configuration.

As described in Dalla et al. (2015), SEPs experience deceleration during propagation through interplanetary space because of adiabatic deceleration and drift effects. In the work
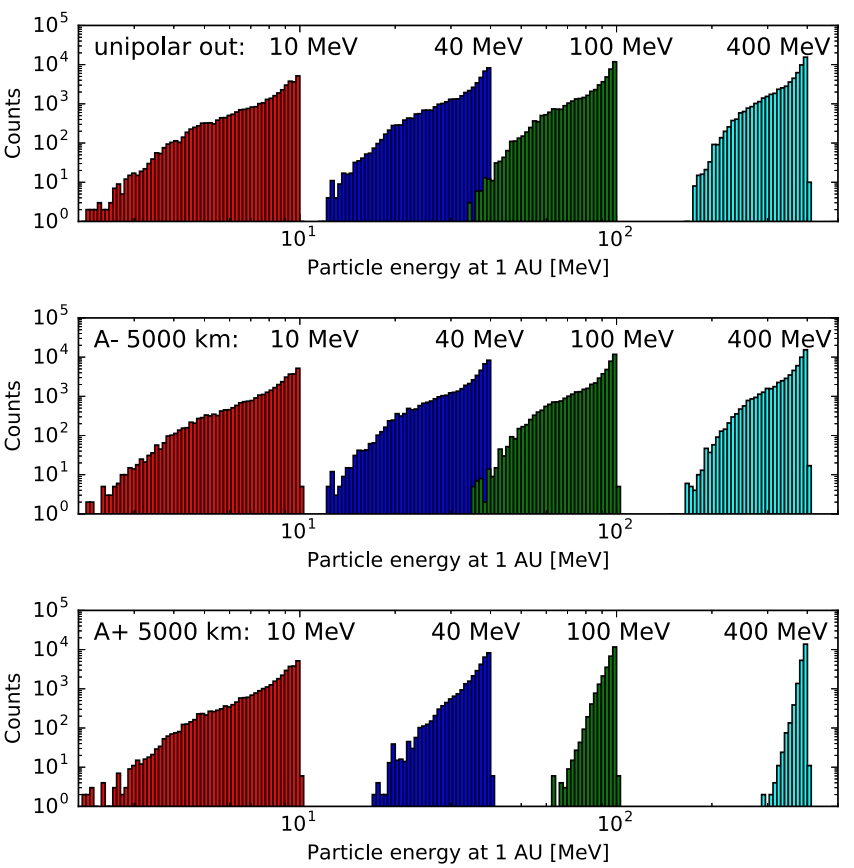

Figure 13. Histograms of measured proton energies at the times of 1 au crossings. Colors depict protons injected at $10 \mathrm{MeV}$ (red), $40 \mathrm{MeV}$ (blue), $100 \mathrm{MeV}$ (green), and $400 \mathrm{MeV}$ (cyan). Top: unipolar field, pointing outward. Middle: HCS thickness scaled to $5000 \mathrm{~km}$ at $1 \mathrm{au}$, with an $A$ - field configuration. Bottom: HCS thickness scaled to $5000 \mathrm{~km}$ at $1 \mathrm{au}$, with an $A$ + field configuration. Deceleration is seen at all energies, but the $A+$ configuration suppresses drifts, and thus, deceleration.

presented in this manuscript, protons have been injected into the simulation at the described energies, with deceleration happening by the time they reach 1 au. Thus, protons that are detected at 1 au as, e.g., $100 \mathrm{MeV}$ protons, will have likely been injected at higher 

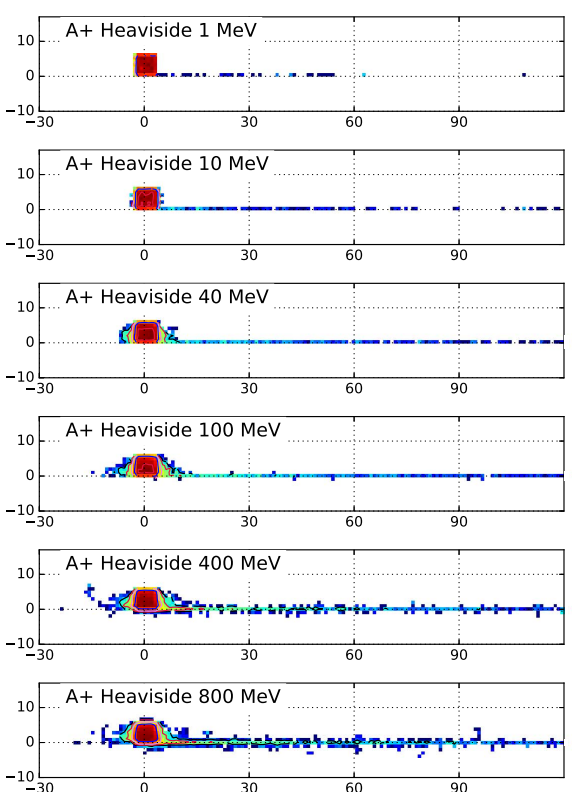
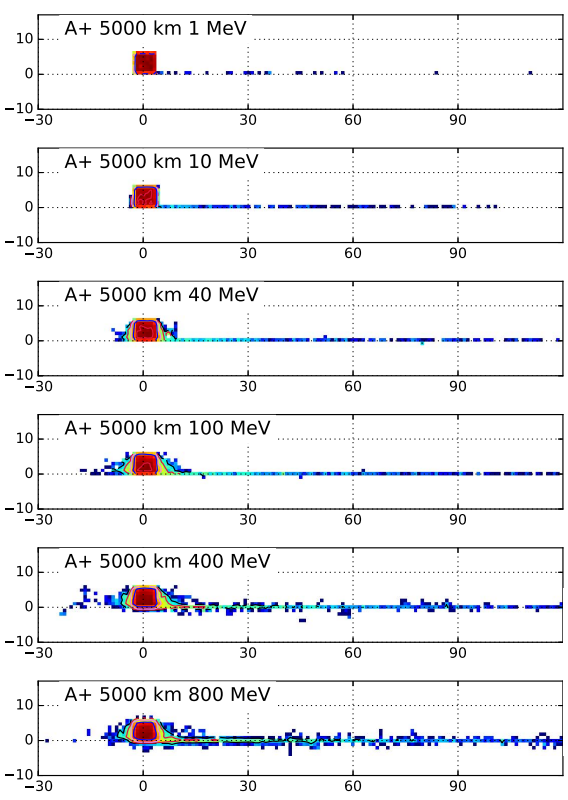
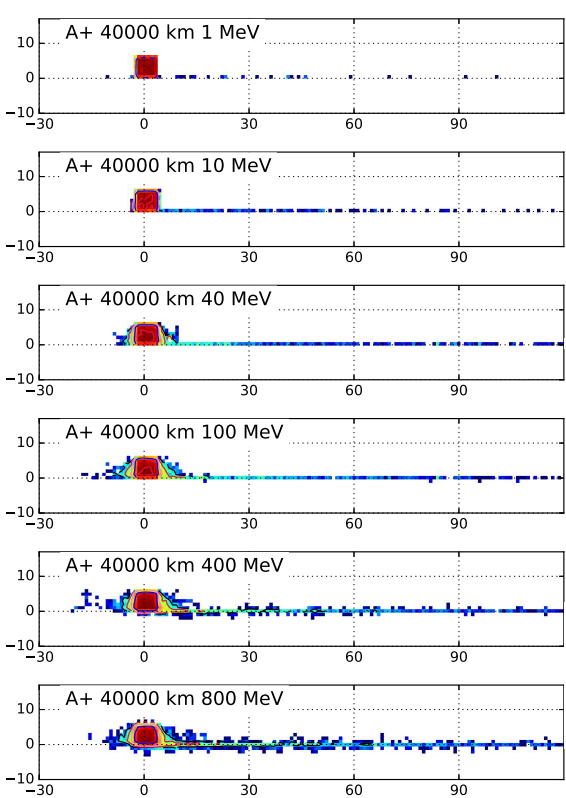

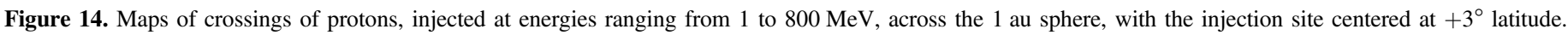

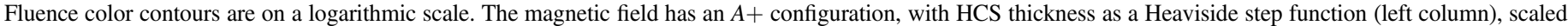

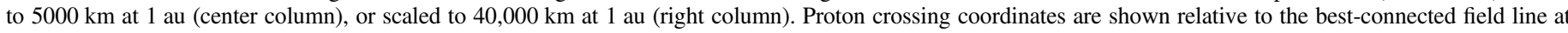

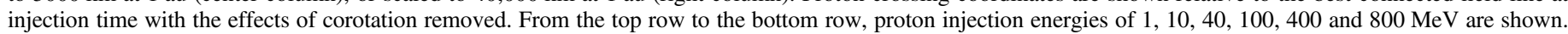
Drift of protons across the HCS is nonexistent at energies below $400 \mathrm{MeV}$.
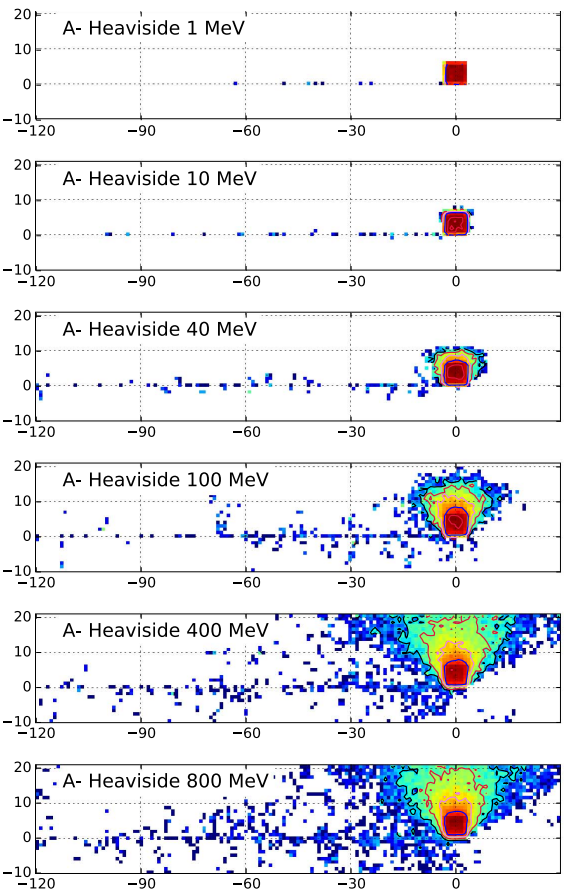
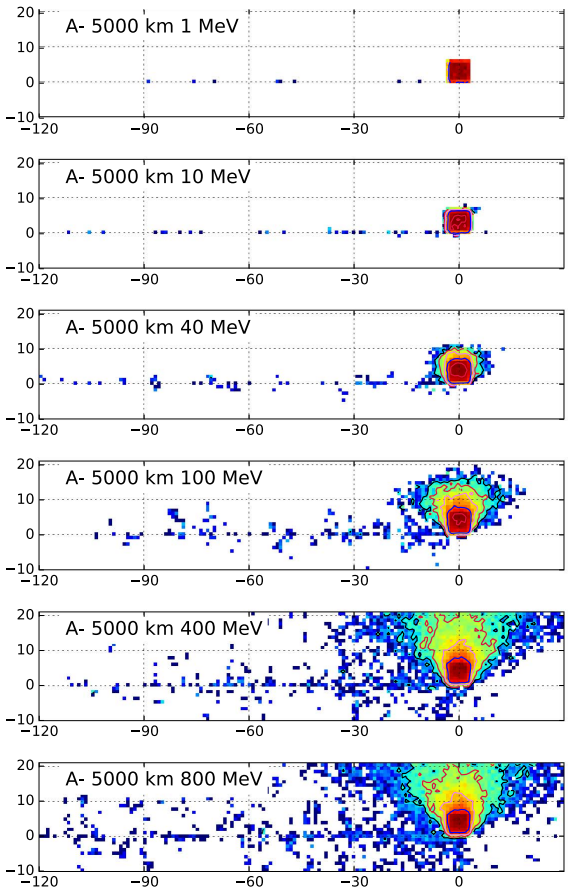
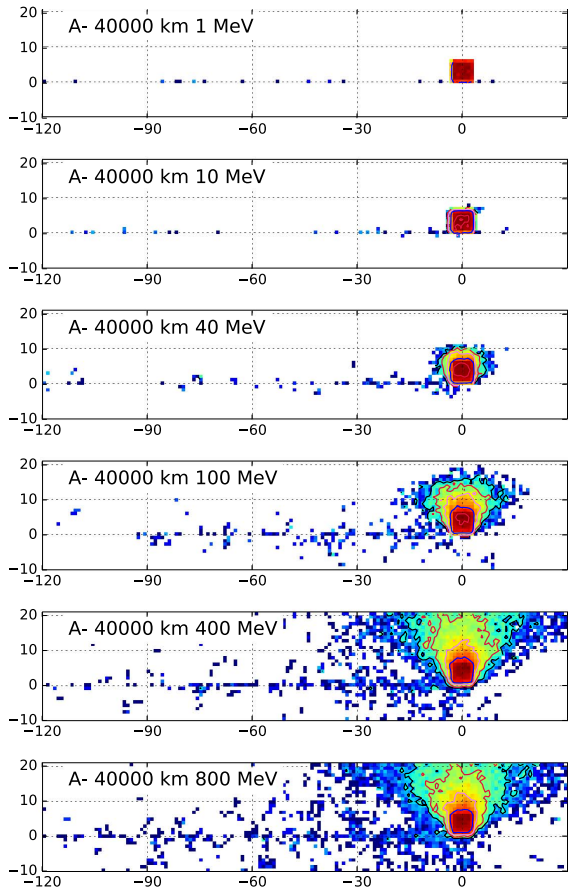

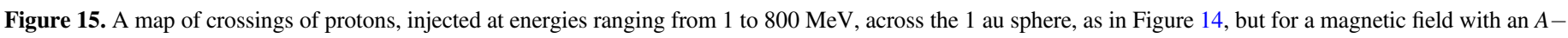

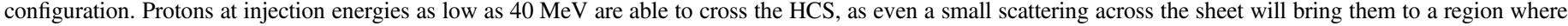
they drift farther away from the sheet. Crossing counts, however, remain low.

energies, and will thus have experienced greater drifts due to the velocity dependencies involved. In Figure 13, we plot histograms of 1 au crossing energies, over the duration of the simulation, for protons injected at energies of $10,40,100$, and $400 \mathrm{MeV}$, for three different magnetic field configurations. For a unipolar outward-pointing IMF, and for an IMF with an $A$ - configuration, protons can decelerate by as much as over $50 \%$ of their initial energy. However, for an $A+\mathrm{IMF}$ configuration, protons are confined to the vicinity of the HCS, and deceleration due to drifts is suppressed to as little as $<25 \%$.

The crossing of SEPs from one IMF polarity to another, across sector boundaries caused by a wavy HCS, is a complex question that we cannot fully analyze within the scope of this work. A first step, however, is to assess the efficiency of particle drifts and scattering in transporting SEPs across a flat HCS. In order to analyze this, we injected protons of six 
different energies $(1,10,40,100,400$, and $800 \mathrm{MeV})$ from a $6^{\circ} \times 6^{\circ}$ angular injection window, centered at $+3^{\circ}$ within an $A+$ configuration. In Figure 14, we plot these results with the effects of corotation removed, for three different current sheet thicknesses. At small energies, only the current sheet drift spreads particles outside the well-connected region, but at energies above $40 \mathrm{MeV}$, some drifts in both latitude and longitude are visible. However, proton energies need to exceed $100 \mathrm{MeV}$ in order to be ejected from the current sheet to the southern hemisphere. For comparison, the Larmor radius of $400 \mathrm{MeV}$ protons at $1 \mathrm{au}$, assuming, for example, a pitch-angle of $\alpha \approx 5^{\circ}$, is of the order of $40,000 \mathrm{~km}$.

In Figure 15, we plot the same crossings as in Figure 14, but for an IMF with a $A-$ configuration. Again, at low energies, the current sheet drift is the primary way particles spread outside the well-connected region. However, as general drift directions are away from the current sheet, any particles that are transported along the current sheet and then scatter away from it can easily propagate farther away from it. Thus, at energies as low as $40 \mathrm{MeV}$, protons are seen to scatter into the southern hemisphere. We note, however, that if the injection region of protons does not coincide with the current sheet, protons within an $A$ - configuration are unlikely to reach the current sheet, and thus unlikely to scatter across it.

Thus, we conclude that an injection event constrained to one hemisphere can, because of lateral drifts and the HCS, remain undetectable in the opposite hemisphere.

\section{Conclusions}

We simulated the propagation of solar energetic protons with energies ranging from 1 to $800 \mathrm{MeV}$ within multiple different IMF conditions, assessing the effects a flat HCS located at the heliographic equator has on proton drifts and propagation. We show that, in the presence of a flat HCS, drifts along the current sheet are significant, allowing high-energy protons to drift over $180^{\circ}$ in longitude. We show that both $A+$ and $A$ - configurations of the IMF allow for significant current sheet drift, which helps protons reach regions far from the injection longitude.

We assessed the effects of current sheet thickness on proton propagation, and found there to be negligible difference between simulations using realistic parameters or a step function. Gradient drifts due to sheet thickness profiles are found to be nonexistent. Thus, we conclude that using a step-mode current sheet is an adequate tool in numerical simulations.

We placed virtual observers at a distance of 1 au from the Sun and generated time profiles mimicking space observations. The IMF configuration was confirmed to significantly affect the qualitative shape of time profiles at different observer locations. For an injection location centered at the HCS, the A+ configuration confined protons to the vicinity of the HCS, whereas the $A$ - configuration caused observers at both northern and southern latitudes to observe particle fluxes. Latitudes separated from the injection region exhibited harder power laws in particle flux compared with latitudes with injection, because of the energy dependence of drifts. The current sheet drift of protons was detectable for an observer at the solar equator, causing an additional abrupt peak early in the event at eastern longitudes for the $A$ - configuration and western longitudes for the $A+$ configuration. In the $A+$ case, this generated dualcomponent time profiles, which could be misinterpreted as being accelerated by two distinct events.
We find that the IMF configuration can affect the extent of deceleration experienced by SEPs, with high-energy particles in an $A+$ configuration retaining a significant portion of their energy due to constraints upon latitudinal drifting extents. When propagating within an $A$ - IMF configuration, latitudinal drifts are not constrained as they are toward the poles, and particles experience deceleration in agreement with the unipolar case. As drift effects are dependent on particle energy, deceleration plays an important role in all of our other results as well. Protons detected at 1 au with a given energy will have been injected at sometimes significantly higher energies, and thus, will have been able to drift greater distances during their interplanetary propagation.

Finally, we assess the motion of particles across a flat HCS, and find that only scattering allows for protons to cross into the opposite hemisphere. The amount of scattering across the HCS is found to be small. Thus, within our model, if a current sheet exists between an injection site and an observer, fluences can be strongly suppressed.

We note that all presented results involve a relatively small injection region, perhaps associated best with a flare without an associated CME. For injection at wider fronts, a tile-based approach like the one presented in Marsh et al. (2015) can be applied.

The configuration of the HCS and its associated drifts have thus been show to play an important role in the longitudinal and latitudinal transport of SEPs, the generation of complex time profiles, hardened proton spectra, and also the absence of flux at field lines close to the best-connected one. The presented results are applicable to eruptions during the solar minimum, when the solar magnetic field resembles a dipole. To model active phases of the Sun, when the HCS is tilted and wavy, resulting in a more complex IMF, additional investigations are required, and these will be the subject of future work.

The authors wish to thank the Leverhulme Trust for providing funding for this research through grant number RPG-2015-094. S.D. acknowledges support from the UK Science and Technology Facilities Council (STFC) (grant ST/ M00760X/1). The authors wish to thank the anonymous referee for insightful comments and suggestions.

\section{References}

Aran, A., Sanahuja, B., \& Lario, D. 2005, AdSpR, 36, 2333

Boris, J. 1970, Acceleration Calculation from a Scalar Potential, Tech. Rep., MATT-769 (Princeton NJ: Plasma Physics Lab.)

Burger, R. A. 2012, ApJ, 760, 60

Burger, R. A., Moraal, H., \& Webb, G. M. 1985, Ap\&SS, 116, 107

Chollet, E. E., Giacalone, J., \& Mewaldt, R. A. 2010, JGRA, 115, 6101

Dalla, S., \& Browning, P. K. 2005, A\&A, 436, 1103

Dalla, S., Marsh, M. S., \& Battarbee, M. 2017, ApJ, 834, 167

Dalla, S., Marsh, M. S., Kelly, J., \& Laitinen, T. 2013, JGRA, 118, 5979

Dalla, S., Marsh, M. S., \& Laitinen, T. 2015, ApJ, 808, 62

Eastwood, J. P., Balogh, A., Dunlop, M. W., \& Smith, C. W. 2002, JGRA, 107,1365

Ebert, D., Musgrave, F. K., Peachey, D., Perlin, K., \& Worley, S. 2003 , Texturing and Modeling: A Procedural Approach (3rd ed.; San Francisco, CA: Morgan Kaufmann)

Guo, X., \& Florinski, V. 2014, JGRA, 119, 2411

He, H.-Q., Qin, G., \& Zhang, M. 2011, ApJ, 734, 74

Jokipii, J. R., \& Levy, E. H. 1977, ApJ, 213, L85

Kelly, J., Dalla, S., \& Laitinen, T. 2012, ApJ, 750, 47

Kóta, J., \& Jokipii, J. 2001, AdSpR, 27, 607

Kubo, Y., Nagatsuma, T., \& Akioka, M. 2009, J. Natl. Inst. Inf. Commun. Technol., 56, 17 http://www.nict.go.jp/publication/shuppan/kihoujournal/journal-vol56no1_2_3_4/journal-vol56no1-4_020102.pdf

Laitinen, T., Kopp, A., Effenberger, F., Dalla, S., \& Marsh, M. S. 2016, A\&A, 591, A18 
Luhmann, J., Ledvina, S., Krauss-Varban, D., Odstrcil, D., \& Riley, P. 2007, AdSpR, 40, 295

Marsh, M. S., Dalla, S., Dierckxsens, M., Laitinen, T., \& Crosby, N. B. 2015, SpWea, 13, 386

Marsh, M. S., Dalla, S., Kelly, J., \& Laitinen, T. 2013, ApJ, 774, 4

Parker, E. N. 1958, ApJ, 128, 664

Pei, C., Bieber, J. W., Burger, R. A., \& Clem, J. 2012, ApJ, 744, 170

Press, W. 1996, Numerical Recipes in Fortran 90 (Cambridge: Cambridge Univ. Press)
Roelof, E. 1969, in Lectures in High-Energy Astrophysics, ed. H. Ögelman \& J. Wayland (NASA SP-199; Washington, DC: NASA), 111

Speiser, T. W. 1965, JGR, 70, 4219

Strauss, R. D., Potgieter, M. S., Büsching, I., \& Kopp, A. 2012, Ap\&SS, 339,223

Turner, R. 2000, ITPS, 28, 2103

Winterhalter, D., Smith, E. J., Burton, M. E., Murphy, N., \& McComas, D. J. 1994, JGR, 99, 6667

Zhang, M., Jokipii, J. R., \& McKibben, R. B. 2003, ApJ, 595, 493 